\title{
Observation of Exclusive Di-Photon Production in Hadron-Hadron Collisions
}

\author{
Erik Brücken* ${ }^{* \dagger}$ \\ Helsinki Institute of Physics \\ and \\ Department of Physics \\ FIN-00014 University of Helsinki \\ Finland \\ E-mail: brucken@cc.helsinki.fi
}

\begin{abstract}
We present the first observation and cross section measurement of exclusive photon pair production in proton-antiproton collisions at $\sqrt{s}=1.96 \mathrm{TeV}$ taken by the Run II Collider Detector at Fermilab. We select events with two electromagnetic showers, each with transverse energy $E_{T}>2.5$ $\mathrm{GeV}$ and pseudorapidity $|\eta|<1.0$, with no other particles detected in $-7.4<\eta<+7.4$. The two showers have similar $E_{T}$ and have $\Delta \phi \sim \pi$. A subset of those events having charged tracks are consistent with the QED process $p \bar{p} \rightarrow p+e^{+} e^{-}+\bar{p}$ by two-photon exchange, giving confidence in the selection methods for the exclusive diphoton events. A subset without any charged tracks are candidates for the exclusive process $p+\bar{p} \rightarrow p+\gamma \gamma+\bar{p}$. Data from wire chambers near electromagnetic shower maximum shows that a possible background from exclusive $\pi^{0} \pi^{0}$ production is consistent with zero, as expected, and other backgrounds are negligible. The events are explained as the double pomeron exchange process (D IP E): IP + IP $\rightarrow \gamma+\gamma$, observed here for the first time. The measured cross section is in agreement with a perturbative QCD calculation. This process is closely related to exclusive Higgs boson production $p+p \rightarrow p+H+p$, that could be observed at the LHC.
\end{abstract}

The 2011 Europhysics Conference on High Energy Physics-HEP 2011,

July 21-27, 2011

Grenoble, Rhône-Alpes France

${ }^{*}$ Speaker.
†On behalf of the CDF Collaboration. 


\section{Introduction}

We present the successful search for the production of exclusive photon-pairs in proton antiproton collisions at the Run 2 Collider Detector at Fermilab (CDF II) [1]. The strong interaction process $p \bar{p} \rightarrow p+\gamma \gamma+\bar{p}$ with vacuum quantum number exchange is one of the cleanest apart from elastic scattering. Here "+" denotes the presence of large rapidity gaps $(\Delta y \gtrsim 4)$. At leading order two direct high- $p_{T}$ photons can be produced via gluon-gluon fusion including another gluon exchange to cancel the color of the fusing gluons. This results in intact outgoing proton/antiprotons and no additional hadrons produced. The theoretically dominant production process is gluon fusion through quark loops: $g g \rightarrow q \bar{q} \rightarrow \gamma \gamma$ [2,3]. To ensure exclusivity no other hadrons are allowed to be produced. The so called "rapidity gap survival factor", $S^{2}$ determined from other diffractive cross sections handles such scenarios. Additional hadrons via gluon radiation are Sudakov suppressed. The probability for the absence of proton/antiproton dissociation so far can only be calculated via Regge theory using the t-channel exchange formalism called pomeron IP (see for example [4]). Perturbative QCD is not applicable due to small $Q^{2}$ and thus large $\alpha_{s}$.

The cross section is predicted [2,3] to be small, $\sigma(\gamma \gamma)_{\text {exclusive }} \sim 0.7 \mathrm{pb}$ for $|\eta(\gamma)|<1.0$ and $E_{T}(\gamma)>2.5 \mathrm{GeV}$ with a claimed factor $\sim 3$ uncertainty. The largest uncertainty factor is due to the skewed, unintegrated gluon distribution function $f_{g}\left(x_{1}, x_{2}, Q^{2}\right)$.

Other known processes can produce an exclusive $\gamma \gamma$ final state. Quark-antiquark annihilation $q \bar{q} \rightarrow \gamma \gamma$ and production via coherently radiated virtual photons $\gamma \gamma \rightarrow \gamma \gamma$ are however heavily suppressed and can be neglected as background to the exclusive QCD process. Considerable backgrounds to exclusive $\gamma \gamma$ events are exclusive $\pi^{0} \pi^{0}$ and $\eta \eta$, with each meson decaying to two photons and the inelastic exclusive production of $\gamma \gamma$ with unseen dissociating proton/antiprotons due to detector coverage limitations.

As a control study we use the exclusive $e^{+} e^{-}$production, a pure QED process that has been observed at CDF [5] with a well measured cross section.

\section{The Experiment}

For this study, data with a total of $1.11 \mathrm{fb}^{-1}$ integrated luminosity of $p \bar{p}$ has been collected at CDF II, at the Tevatron with $p \bar{p}$ collisions at $\sqrt{s}=1.96 \mathrm{TeV}$. The CDF II detector is a general purpose detector [1]. This solenoidal detector is approximately azimuthal and forward-backward symmetric. Surrounding the beam pipe is a tracking system consisting of a silicon microstrip detector, a cylindrical drift chamber (COT) [6], and a solenoid providing a 1.4 Tesla magnetic field. The tracking system is fully efficient at reconstructing isolated tracks with $p_{T} \geq 1 \mathrm{GeV} / \mathrm{c}$ and $|\eta|<1$. It is surrounded by the central and end-plug calorimeters covering the range $|\eta|<3.6$. Both calorimeters have separate electromagnetic and hadronic compartments. A proportional wire chamber (CES) [7] is embedded in the central EM calorimeter, $|\eta|<1.1$, at a depth of six radiation lengths. The forward region at $3.6<|\eta|<5.2$ is covered by a lead-liquid scintillator calorimeter called the Miniplug [8]. At higher pseudorapidities, $5.4<|\eta|<7.4$, scintillation counters, called beam shower counters (BSC), are located on each side of the CDF detector. Gas Cherenkov detectors, with 48 photomultipliers per side, covering $3.7<|\eta|<4.7$, determine the luminosity with a $6 \%$ uncertainty [9], and were required in this study to be empty. 
The data was recorded using a dedicated trigger. At the hardware level (L1) the trigger requires one EM shower with $E_{T}>2 \mathrm{GeV}$ and $|\eta|<2.1$ and a veto on BSC-1 counters $(|\eta|=5.4-5.9)$, filtering for rapidity gaps. At higher level two EM showers are required and the trigger imposed a Hadronic:EM ratio $<0.125$, isolation cuts and a $\chi^{2}$ requirement on the shape of the shower. The strong BSC1 veto rejects most inelastic collisions and events with pile-up.

\section{Analysis and Results}

We expect from the central exclusive produced photon-pair events to have two isolated EM showers in the central detector and a very forward outgoing proton and antiproton. Protons and antiprotons are far too forward to be detectable at CDF II. We select events with two good central EM showers and no other particles in the full detector. As already mentioned we use the well measured exclusive $e^{+} e^{-}$production process as a justification for our analysis methods. Electrons and photons are at the detector level similar except that electrons leave tracks. Each EM shower is required to have an $E_{T}>2.5 \mathrm{GeV}$ to avoid trigger threshold inefficiencies and has to be within $|\eta|<$ 1.0. Next we filter for exclusivity by selecting only those events that show clear rapidity gaps. After studying the noise level of the calorimetry and the BSC's using random data triggered on bunch crossing times (zero-bias), we search for any activity above noise level except our signal. Using the zero-bias data we can show that the efficiency of selecting exclusive events is $6.8 \% \pm 0.4 \%$ (syst). Subsequently we apply quality cuts on the remaining events to ensure good photons or electrons. We are left with a sample of 81 clean events holding two EM showers. We found that 34 had exactly two oppositely charged tracks, 43 had no tracks in the COT, and four were in neither class. The event sample with two tracks show a good agreement with the earlier observed QED process $p \bar{p} \rightarrow p+e^{+} e^{-}+\bar{p}$ [5]. The measured cross section of $2.88_{-0.48}^{+0.57} \pm 0.63$ pb compares well with the theoretically predicted of $3.25 \pm 0.07 \mathrm{pb}$. The sample consisting of 43 events clean of any tracks

Table 1: Summary of parameters for the measurement of the exclusive photon-pair cross section for a $E_{\mathrm{T}}>$ $2.5 \mathrm{GeV}$ and $|\eta|<1.0$.

\begin{tabular}{lc}
\hline \hline Exclusive $\gamma \gamma$ & Value \\
\hline Events & 43 \\
Integrated luminosity $\mathscr{L}_{\text {int }}$ & $1.11 \pm 0.07 \mathrm{fb}^{-1}$ \\
Photon pair efficiency & $0.40 \pm 0.02$ (stat) \pm 0.03 (syst) \\
Exclusive efficiency & $0.068 \pm 0.004$ (syst) \\
Probability no conversions & $0.57 \pm 0.06$ (syst) \\
$\pi^{0} \pi^{0}$ background (events) & $0.0,<15(95 \%$ CL.) \\
Dissociation background (events) & $0.14 \pm 0.14$ (syst) \\
\hline \hline
\end{tabular}

shows good kinematical agreement with the theoretical expected exclusive $\gamma \gamma$ production [10]. The invariant mass $M_{\gamma \gamma},|\pi-\Delta \phi|$ and the 2-vector sum of $E_{T}$ are shown in Fig. 1, in comparison with the signal Monte Carlo, all normalized to the same number of events as the data. Whether the 43 events are not pure $\gamma \gamma$ but contaminated with $\pi^{0} \pi^{0}$ events is a key-point of this study. Note, $\gamma \pi^{0}$ is forbidden by C-parity. The $\pi^{0}$ decays mostly into two photons with an minimum opening angle of $\Delta \Theta_{\min }=2 \tan ^{-1}\left(\frac{m(\pi)}{p(\pi)}\right)=3.1^{\circ}$ for $p(\pi)=5 \mathrm{GeV}$. Having well separated photons from the $\pi^{0}$ decay, we can make use of the CES chambers to distinguish between $\pi^{0}$ and $\gamma$. A $\pi^{0}$ can 

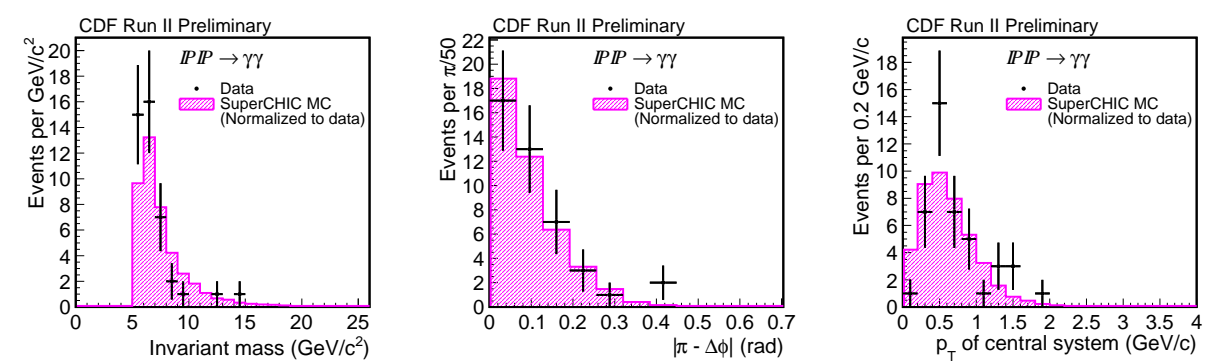

Figure 1: Two-photon candidates: (left) Invariant mass distribution (middle) $|\pi-\Delta \phi|$ distribution (right) $p_{T}$ distribution of the central system. All error bars are statistical. The MC predictions are normalized to data.

be seen as a fake photon if one photons falls into an inactive detector region (8\%) or ranges out before reaching the wire chambers. We sum the number of reconstructed CES showers of both EM objects of the event and compare it with the hypothesis of having only background or signal. It favors strongly the $\gamma \gamma$ signal simulation and disagrees with the $\pi^{0} \pi^{0}$ simulation. The best fit to the composition of signal and background gives a fraction $f\left(\pi^{0} \pi^{0}\right)$ of 0.0 , with a $95 \%$ C.L. upper limit of 15 events. A recent theoretical calculation [11] predicts $\sigma_{\text {excl }}\left(\pi^{0} \pi^{0}\right)=6-24 \mathrm{fb}$ for $E_{T}\left(\pi^{0}\right)>2.5 \mathrm{GeV} / \mathrm{c}$ and $|\eta|<1.0$, much smaller than our measured cross section. Exclusive $\eta \eta$ production is also expected to be negligible. The only other significant background could be undetected proton dissociation, about $10 \%$ for the QED $e^{+} e^{-}$process but $<1 \%$ for IP $+\mathrm{IP} \rightarrow$ $\gamma+\gamma$. Taking into account trigger, reconstruction, identification and conversion efficiencies as well as the exclusivity efficiency (see Table 1 ) we obtain a cross section of $\sigma_{\gamma \gamma, \text { excl }}\left(|\eta|\left\langle 1, E_{T}(\gamma)\right\rangle\right.$ $2.5 \mathrm{GeV})=2.48_{-0.35}^{+0.40}(\mathrm{stat})_{-0.51}^{+0.40}(\mathrm{syst}) \mathrm{pb}$. Our result is in good agreement with presently the only theoretical calculation [3].

\section{Conclusions}

We have observed for the first time the exclusive production of two high- $p_{T}$ photons in hadron hadron collisions showing a good agreement with theoretical predictions. This gives hints of exclusive Higgs boson production $[2,12] p+p \rightarrow p+H+p$, that might be detectable at the LHC. The dominant hard process $g g \rightarrow \gamma \gamma$ via a light quark loop can be exchanged by a heavy top-quark loop in $g g \rightarrow H$ with $H$ being a light SM or SUSY Higgs boson. If Higgs exists the central exclusive production of the Higgs at LHC must be possible.

\section{References}

[1] D. Acosta et al. (CDF Collaboration), Phys. Rev. D 71, 032001 (2005) and references therein; D. Amidei et al. (CDF Collaboration), Nucl. Instrum. Methods 350, 73 (1994); F. Abe et al. (CDF Collaboration), Phys. Rev. D 50, 2966 (1994).

[2] V. A. Khoze et al., Eur. Phys. J. C 38, 475 (2005).

[3] L. A. Harland-Lang, V. A. Khoze, M. G. Ryskin, and W. J. Stirling, Eur. Phys. J. C 69, 179 (2010).

[4] S. Donnachie, G. Dosch, P. V. Landshoff and O. Nachtmann, "Pomeron Physics and QCD," Cambridge University Press (2002). 
[5] A. Abulencia et al. (CDF Collaboration), Phys. Rev. Lett. 98, 112001 (2007).

[6] A. Affolder et al. (CDF Collaboration), Nucl. Instrum. Methods Phys. Res. Sect. A 526, 249 (2004).

[7] L. Balka et al., Nucl. Instrum. Methods A 267, 272 (1988).

[8] M. Gallinaro et al., IEEE Trans. Nucl. Sci. 52, 879 (2005).

[9] D. Acosta et al., Nucl. Instrum. Methods A 494, 57 (2002).

[10] SUPERCHIC, http://projects.hepforge.org/superchic/

[11] L. A. Harland-Lang, V. A. Khoze, M. G. Ryskin, and W. J. Stirling, Eur. Phys. J. C 71 (2011) 1714

[12] V. A. Khoze, A. D. Martin, and M. G. Ryskin, Eur. Phys. J. C 14, 525 (2000). 